\title{
A PILOT STUDY: DESIGNING and TESTING THE TASK PARAMETERS
}

\author{
Meltem Ballan ${ }^{\mathrm{a}}$
}

The aim of this study was to test the validity, efficiency and measurements of a two-digit number comparison study. The particular task was tested by six participants. The study examines the possible influence of visual quality judgment on quantity judgments of numbers. Therefore, the visual representation of stimulus as well as the efficiency of fixation tested. The duration of the task and repetition of stimuli were designed using the participants' feedback as well as some basic statistical tests. Our preliminary data suggested that a task consisted of six blocks of 72 trials in each will be efficient to test our study question on the relationship between visual quality and number perception. Our preliminary results suggested that the visual quality affects the participants' behavior and brain dynamics. We are currently working on different analysis techniques to finalize our actual study.

Keywords: Pilot, design parameters, two-digit number perception, ERP

\section{Introduction}

Human perception consists of two main processes, sensory input (e.g., visual, tactile, auditory) processing and cognitive processing (e.g., quantity). In this context, the quantitative perception of visual sensory input has been used to understand the underlying mechanisms of number perception. The unattended visual attributes of the stimulus presentation (e.g., luminance, size) automatically changes the quantitative perception of the attended stimuli (Tzelgov, 1997; Tzelgov, Yehene, Kotler, \& Alon, 2000). The size congruity effect is one of the common examples of the influence of the physical size of a numerical stimulus on the perceived quantity of the number (i.e., distance effect). The distance effect is defined by logarithmically increasing reaction time (RT) function whereas the size congruity effect is defined as the interactive relationship between the physical size of the stimulus and the quantity. For example, the response time for comparing paired numbers presented as a larger number quantity with larger font and a smaller number quantity with smaller font will be faster than comparing larger number quantity by smaller font and smaller number quantity by larger font. Both distance and size congruity effects show that the sizes or quantities conveyed by numbers are processed even when completely irrelevant to the task at hand or even when they clearly interfere with processing the attended stimulus. This suggests that numerals are assigned to their internal representations instantly without any need for conscious processing.

In their seminal paper, Moyer and Landauer (1967) suggested that the spatial distance between two numbers on the mental number line impacts subjects' RTs. For example, the reaction time to decide whether the number 6 is larger than 5 is longer than that required to decide the relative quantity of 9. The well-known name for this phenomenon is the distance effect. In the same article, Moyer and Landauer (1967) suggested that the distance between two numbers is judged similarly to how the distance between two physical stimuli is judged (e.g., format of line and length of the line). Moyer and Landauer (1967) explored the nature of the process involved in adults' choice of a larger number by examining the time that the process requires. Stimuli consisted of two 1-digit numbers $2.5 \mathrm{~cm}$. apart on a white background. Participants pressed the left or right of two switches depending on the placement of the larger number. Results showed that decision time was approximately a linear inverse function of the numerical differences between the 2 stimulus digits. It is suggested that this process is analogous to the process involved in judgments of inequality for physical continua. Many researchers agree with the finding of Moyer and Landauer (1967) regarding single digit numbers (Campbell, 1994; Cipolotti, Warrington, \& Butterworth, 1995; Dehaene, 1992; Dehaene \& Akhavein, 1995; McCloskey, Caramazza, \& Basili, 1985; McCloskey, Macaruso, \& Whetstone, 1992; Noel \& Seron, 1995).

With two-digit numbers, the underlying mechanisms seem to change. In parallel to the single digit number comparison studies, the two-digit number perception (i.e., the distance effect) is influenced by the physical presentation of the stimulus (Ganor-Stern, Tzelgov, \& Ellenbogen, 2007). However, it seems that two-digit numbers may not be processed as one whole because both components of the number (the ones-place and the tens-place) are taken into account when exercising a comparative judgment (Kaufmann et al., 2005; Nuerk, Weger, \& Willmes, 2001). This result suggested that making a decision of whether or not 72 is larger than 65 may take longer than deciding if 76 is larger than 65 because in 72 the tens-place is larger than the reference number. Thus, it is insufficient to process a tensplace digit (e.g., 7) and make a decision about the numeric quantity without evaluating the second digit (i.e. 2 vs. 6) in making a judgment on quantity.

In addition to the studies on number quantity, the sensory quality of two-digit numbers has been also studied. One of the well-studied visual attributes is the notation of number stimuli (Arabic (32) vs. verbal (thirty two) numbers) and its effects on quantity (distance) judgment (Pinel, Dehaene, Riviere, \& LeBihan, 2001). Pinel et al. (2001) studied the effect of verbal versus Arabic formats on a twodigit number comparison task during a combined event related potential (ERP) and functional magnetic resonance 
imaging (fMRI) study. The bilateral extrastriate cortices and a left precentral region were more activated during verbal number than during Arabic number stimulation, while the right fusiform gyrus and a set of bilateral inferoparietal and frontal regions were more activated during Arabic number than during verbal number stimulation. These results support the assumption of a central semantic representation of numerical quantity that relies on a common parietal network shared across notation styles(Pinel, et al., 2001).

In the same context, Cohen Kadosh \& Henik (2006) studied the effects of visual attributes on number perception controlling the luminance parameter of the visual stimulus. The latter was an alternative study to the traditional approach in size congruity studies (Cohen Kadosh, Cohen Kadosh, Kaas, Henik, \& Goebel, 2007; Foltz, Poltrock, \& Potts, 1984; Henik \& Tzelgov, 1982; Szucs \& Csepe, 2005; Tzelgov, Henik, \& Berger, 1992) which suggested an interaction between size congruity and the numerical quantity as luminance. The behavioral results showed that the stimulus quantity was changed by the luminance of the number stimuli. Consequently, Cohen-Kadosh et al. (2007) observed parietal brain region activities were affected by the luminance of the number stimuli. In interpreting this finding, Cohen-Kadosh et al. (2007) reported the parietal lobe might be equipped with neuronal substrates for magnitude processing even for nonspatial dimensions. In other words, the parietal lobe might be involved in the processing of visual qualities along with quantities of the number stimuli.

\section{Aims and Objectives of the Study}

The studies reviewed above have examined the format effect on number perception (i.e. Arabic vs. verbal numbers, lines vs. Arabic numbers), size congruity (i.e. small numbers in smaller font vs. larger numbers in larger font or vice versa), as well as other types of congruity such as the luminance effect (i.e. darker numbers smaller vs. lighter numbers larger or vice versa). In general, these studies concluded that number quantity judgment (e.g., distance effect) can be perturbed by visual or sensory representation of the numbers (e.g., visual noise manipulation). The present study attempted to advance this research further by addressing the question of how the numerical judgment of two-digit numbers (distance effect) is influenced by visual manipulation when they are compared to a memorized reference number (e.g., 65). This study examined whether visual noise could modulate two-digit comparative judgments. Determining the timing and neuronal organization of certain cognitive processes and isolating the specific time window along with the brain regions from the whole process provided information about the effects of visual quality judgments on quantitative judgment of number stimuli.

The analysis of ERPs from high-resolution electroencephalogram (EEG) recordings is a precise method for determining the timing and neuronal organization of specific cognitive processes, especially when no overt behavior can be registered (e.g., when humans plan and prepare for upcoming actions). A common approach to understand underlying brain dynamics of cognitive processes is to analyze positive and negative ERP components. By definition, a component may occur at different times under different task conditions, as long as it arises from the same module and represents the same cognitive function. The scalp distribution and polarity of a component may also vary according to this definition, since the same cognitive function may occur in different parts of a cortical module under different conditions. Ambiguities in interpreting ERP components are the result of task-dependent variations. The latter causes the misinterpretations of certain brain dynamics.

In this study, parietal electrodes were isolated for the analysis $600 \mathrm{~ms}$ after the stimulus onset to determine the relationship between visual quantity and quality factors. The latencies, amplitudes, and inter-hemispheric correlations were described within and between visual quantity and quality factors.

In addition, well-characterized ERP components were focused over parietal brain regions. The following sequence of posterior ERP components have been typically used for number comparison studies: (1) a visual attention stage (P1 component) which was suggested as the attentional influences on sensory selection over occipital regions (Dehaene, 1996; Pinel, et al., 2001; Turconi, Jemel, Rossion, \& Seron, 2004); (2) a visual identification stage (that occurred as N1), which was affected by stimulus notation and occurred bilateral (for Arabic numerals) over occipito-temporal regions (Dehaene, 1996; Turconi, et al., 2004); (3) a magnitude comparison stage, affected by the distance between the reference number and the stimuli, and occurring over both parieto- and occipitotemporal regions on the $\mathrm{P} 2 \mathrm{p}$ and $\mathrm{N} 2$.

All in all, the design parameters become very critical to acquire reliable behavioral and brain signal data. Therefore, we validated our task parameters. The focus of first two experiments was to understand the behavioral basis of the design parameters. The final task was tested in a dimly lit EEG recording room that was identical to the actual task run. Fifteen participants are very common in the related literature (Dehaene, 1996; Pinel, et al., 2001). Therefore, we tested our design parameters with six subjects and recruited fifteen participants for our actual studies.

\section{Materials and Methods}

\section{Subjects}

Six right-handed male subjects between the ages of 18 and 30 participated in the pilot experiments. After positioning the EEG electrode cap on the scalp, the participant was seated in a dimly lit room and responded to test stimuli by pressing one side of a two-sided button box, the left hand side button for numbers smaller than 65 , or the right hand side button for larger numbers. All the subjects signed written consents approved by University of Groningen Human Research Committee.

\section{Tools}

Number stimuli were presented in a simple and wellknown format of Arabic Arial Narrow, font size of 48 and slide size of $256 \times 256$. As all the participants were familiar with the representation format, it was assumed that there was no attentional format bias among the tested individuals.

A MATLAB toolbox (The MathWorks, Natick, MA) was used to produce two different levels of "salt and pepper noise" which consists of randomly distributed dots. Three visual quality levels were defined by noise density parameters that were chosen to limit the visibility of the 
number $(\mathrm{d}=0, \mathrm{~d}=.60, \mathrm{~d}=.75)$. Stimuli were presented
electronically using the E-Prime 2.0 software (Psychology Software Tools, Pittsburgh, PA).

\section{Procedure of the Pilot Behavioral Task}

The task started with an instruction slide, which terminated after $30 \mathrm{~s}$. The subjects were asked to memorize the number 65 as the reference number and then compare the presented numbers with 65 . The reference number was never presented during the task and subjects were reminded several times before the task and an instruction slide included a sentence informing the subject that 65 would not be presented. The visual manipulation levels and factor were neither explained nor included in the instruction. As shown in Figure 1 , the first pilot study began with a fixation cross, followed by a number stimulus displayed until the response button was pressed. Trials were separated by inter-stimulus intervals lasting 3 to 5 s into a block of 72 trials (Pinel, et al., 2001). The selected numbers and manipulation factors were shown in Table 1.

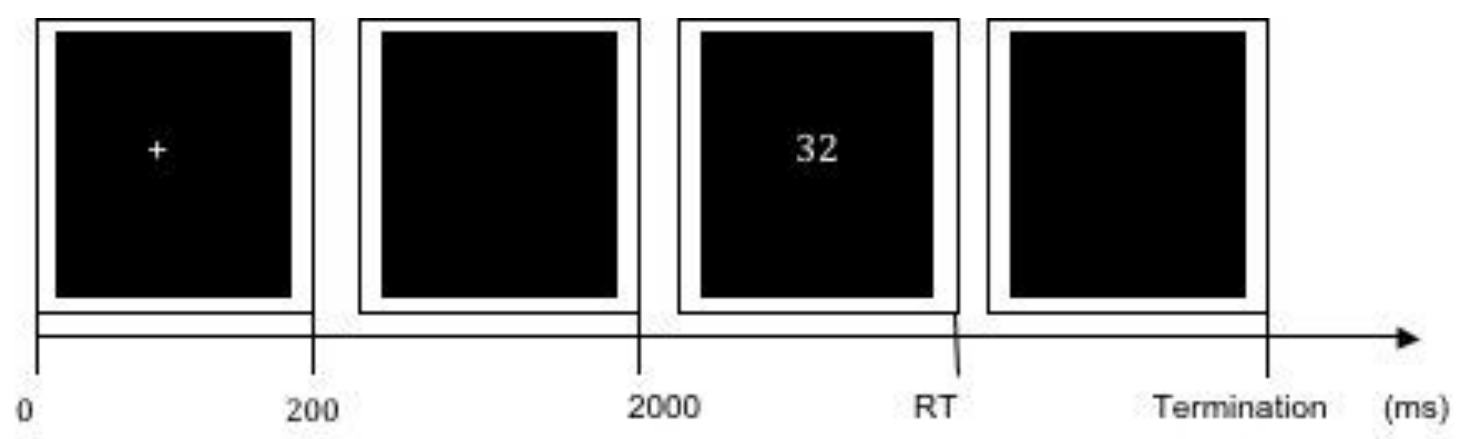

Figure 1: A single trial representing the first reaction time task. The trial starts with a fixation cross lasting $200 \mathrm{~ms}$ followed by a blank screen shown for $2000 \mathrm{~ms}$. The stimulus disappears as soon as the response (i.e., RT) is provided. The termination of an interstimulus interval was calculated as $800 \mathrm{~ms}-\mathrm{RT}<$ Termination $<2800 \mathrm{~ms}-\mathrm{RT}$.

Table 1: The number stimuli chosen among the numbers between 31 and 99 .

\begin{tabular}{lllll}
\hline Distance & $<\mathbf{6 5}$ & $>\mathbf{6 5}$ & Conditions & Noise Conditions \\
\hline Close & $64-63-62-61$ & $69-68-67-66$ & smaller, larger & no, medium, dense \\
\hline Medium & $51-52-54-56$ & $78-77-76-74$ & smaller, larger & no, medium, dense \\
\hline Far & $32-38-41-48$ & $98-92-90-82$ & smaller, larger & no, medium, dense \\
\hline
\end{tabular}




\section{Preliminary Results and Comments}

\section{Results I}

The errors are shown in Table 2. Fixation cross and blank slides were used between two stimuli.

Table 2: Error rate.

\begin{tabular}{cc}
\hline Participant & Error Rate \\
\hline 1 & $8.3 \%$ \\
\hline 2 & $5.5 \%$ \\
\hline 3 & $5.5 \%$ \\
\hline 4 & $11 \%$ \\
\hline 5 & $4.2 \%$ \\
\hline 6 & $8.3 \%$ \\
\hline Average & $7.1 \%$ \\
\hline
\end{tabular}

\section{Comment I}

The first behavioral task consisted of only one block (included 72 trials) to test the fixation parameter. The RTs and the participant's feedback suggested that using a fixation cross was confusing. Participants reported a tendency toward adding the number stimuli rather than simply using the fixation cross to maintain gaze. The participants also reported that the blank slides between two stimuli were also distracting the gaze away from the center of stimulus slide. Therefore, the task was modified to exclude the blank slides and exchange the fixation cross for a fixation asterisk. The modified task was presented to the same six subjects. Our further studies showed the repetition of the task did not affect the accuracy and speed of the task (Ballan, 2011).

\section{Results II}

The average error rate of the task without blank slides and with the fixation asterisks decreased for the second task design (5.3\%); however, the decrease was not statistically significant. The error rates were the total number of errors recorded from each participants.

\section{Comment II}

The task accuracy was increased in the modified design after removing blank slides and exchanging fixation crosses to the asterisks. In order to calculate the statistical significance, paired sample test was applied on the averaged errors of each participant as well as the average errors of all participants. The participants suggested that the task, consisted of 72 trials of a block, was too short to evaluate the unattended visual manipulation and to compare the number stimuli with the memorized reference number 65 . Therefore, the final task was designed consisting of six blocks. Each block had 72 trials and between two blocks with a $30 \mathrm{~s}$ rest interval. We hypothesized that increasing number of stimulus would decrease the error rates.

\section{Final Task Design}

The final task consisted of a short practice block with instructions, followed by six blocks. In the beginning of the task, a short instruction slide appeared for $30 \mathrm{~s}$, then a practice block with 4 stimuli ran for $15 \mathrm{~s}$, followed by six blocks each with 72 stimuli with random inter-stimulus interval (ISI) time between 3 and $5 \mathrm{~s}$ and stimulus duration (SD) terminated by an answer given by the participant for at most $2 \mathrm{~s}$. After each block of $288 \mathrm{~s}$, there was a $30 \mathrm{~s}$ break to prevent fatigue. Figure 2 illustrates the temporal presentation of the stimuli; high peaks of the steps represent the stimulus and the lines were fixation periods between two stimuli. The task was tested on the same six subjects. The accuracy and speed of RTs did not change during the actual study (Ballan, 2011). Therefore, the repetition of task did not interfere with the skills of subjects. We suggest that the practice block decreased the learning effect. 


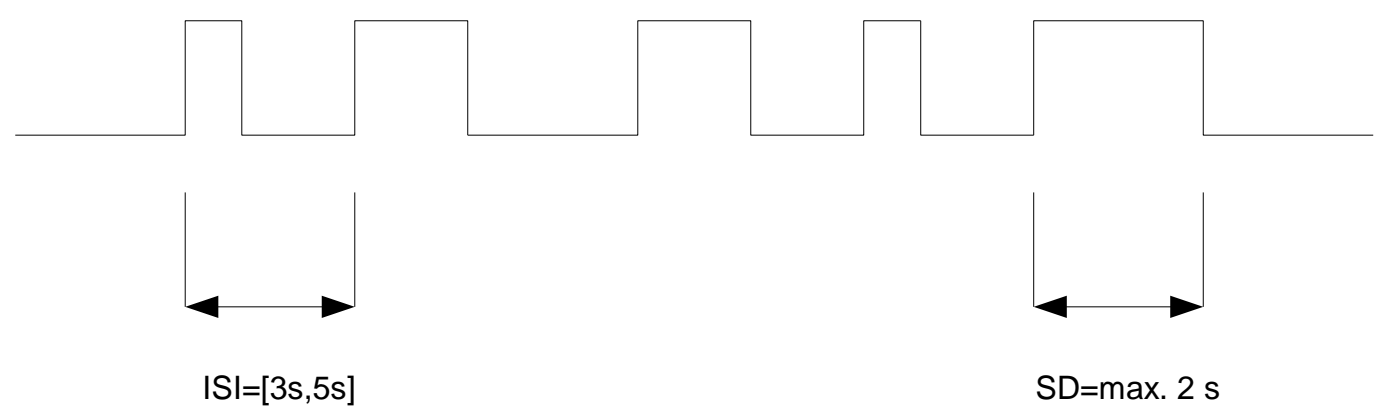

Figure 2: Final task design. Inter-stimulus intervals started as fixation asterisks (shown as the high peaks) followed by the stimulus, which was set to disappear as soon as the button press signal was received. The stimulus duration was selected at a maximum of $2000 \mathrm{~ms}$. and it automatically disappeared if there was no response within this period.

\section{Results and Discussion of Pilot Study}

\section{Behavioral Results and Discussion}

The RT results of six pilot subjects are shown in the Figure 3 and Figure 4. These figures represent the distance and noise patterns of RTs for each block. Figure 3 shows the RTs for different distance conditions without separating the noise conditions whereas Figure 4 shows the RTs for noise condition without considering distance conditions. The patterns showed that distance effect was not pronounced between medium and far distance conditions. The noise pattern showed that there is a consistent RT increase with increasing noise condition.

The block-wise analysis was employed to develop a final task consisting of sufficient trial numbers to provide a sufficient sample size for statistical analysis. In addition, it was important to avoid too long of a task to provide consistent RTs over the trials. The results of paired sample test showed that the numbers of averaged errors between and within subjects were significantly decreased $(\mathrm{p}<0.05)$. Although the rate of errors was very low (total 2620 trials for six subjects 317 errors); the errors were observed more frequently in the first and the last blocks.
Repeated measures of ANOVA test was employed to analyze the block, distance and visual quality effects as well as the interactions. There was no significant effect of block $(\mathrm{F}<1, \mathrm{p}>0.05)$. The results showed a significant main effect of visual quality $(F(2,10)=24$, $\mathrm{p}<0.05)$. Post hoc analysis showed that the significant difference was pronounced for all three noise conditions (i.e., dense $\mathrm{x}$ medium $\mathrm{F}(1,5)=27, \mathrm{p}<0.05$, medium $\mathrm{x}$ no-noise $F(1,5)=12, p<0.05)$. The main distance effect was also significant $(\mathrm{F}(1,10)=25.8, \mathrm{p}<0.05)$. There was no significant difference between medium and far distance conditions $(\mathrm{F}<1, \mathrm{p}>0.05)$; however, the difference between close and medium distance conditions was significant $(\mathrm{F}(1,5)=25.7, \mathrm{p}<0.05)$. Second step of analysis was decreasing the number of parameters. Since the significant difference was not reported between blocks as well as the medium and far distance conditions we averaged the RTs over the blocks and medium and far distance levels. The standard deviation decreased by increasing distance condition and decreasing noise level. Figure 5 summarizes the average RT patterns with $1 \%$ error bars. 


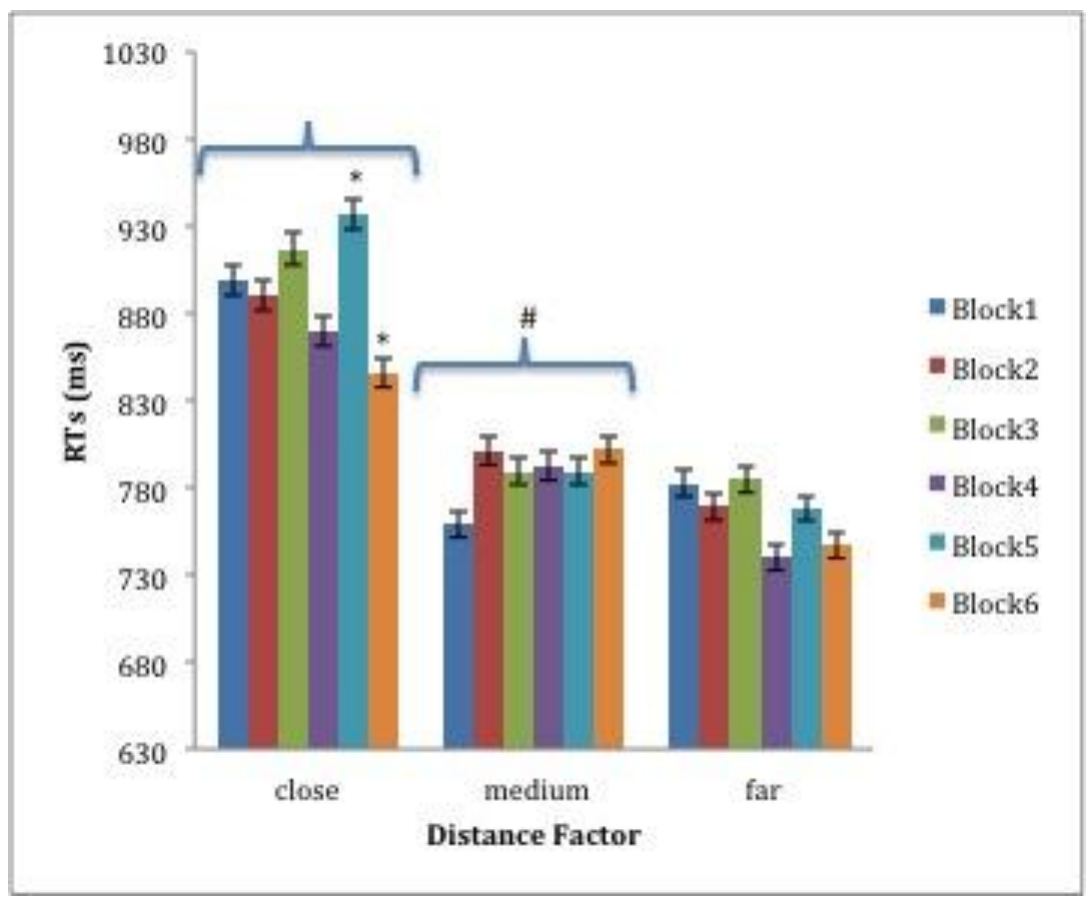

Figure 3: Block-wise distance effect on RT (in ms). The error bars represent the $1 \%$ of the error amount. The mean RTs were calculated for each block for six pilot participants. The mean RTs for close distance numbers were longest for each block whereas the RTs did not show a consistent trend for medium and far distances (i.e. the RT for first block is fastest for medium; the RT of last block was fastest for far distance). The latter indicated an interaction between distance and visual quality.

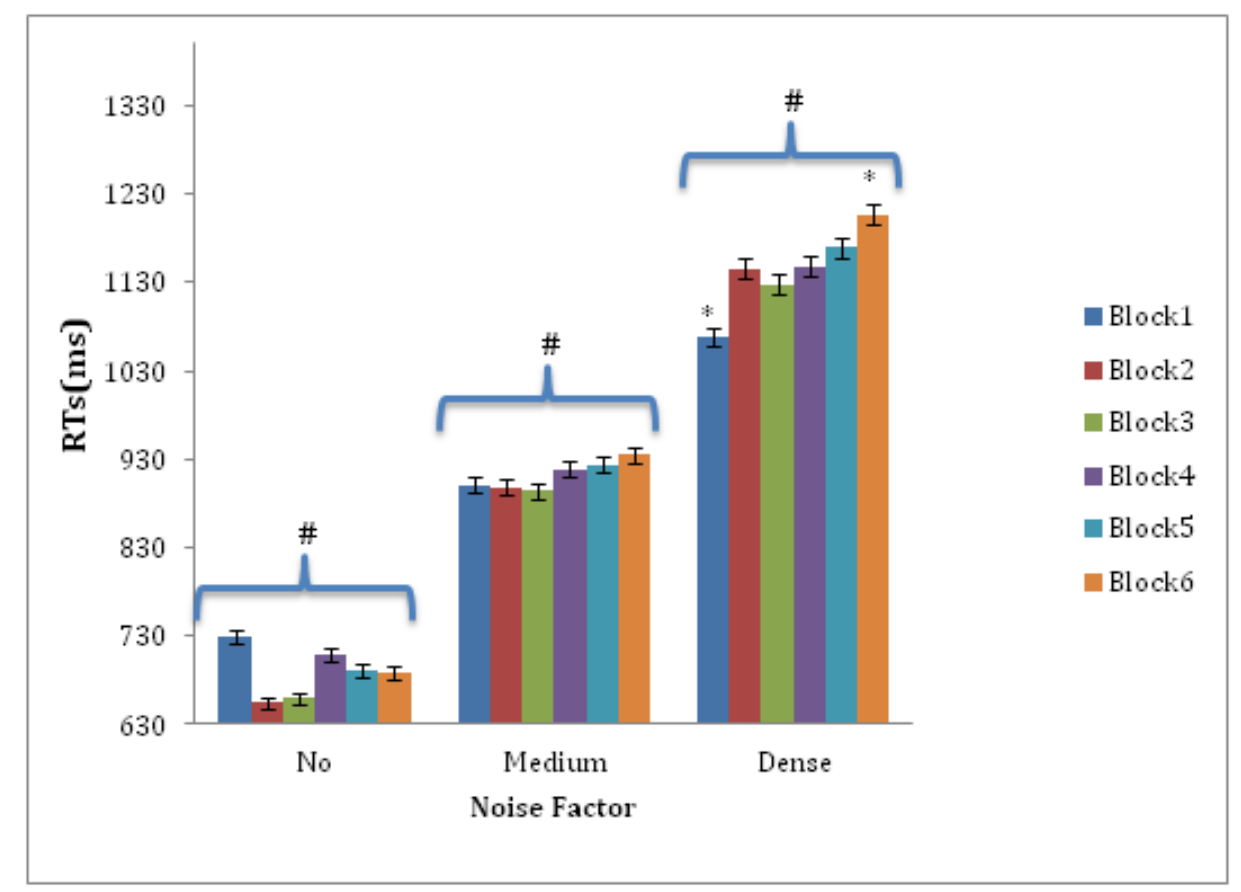

Figure 4: Block-wise noise effect on the RT (in ms). The error bars represent the $1 \%$ of the error amount. The RTs showed similar trends for all blocks. The fastest mean RTs were recorded for no-noise added stimuli whereas the longest RTs recorded for dense noise added stimuli. 


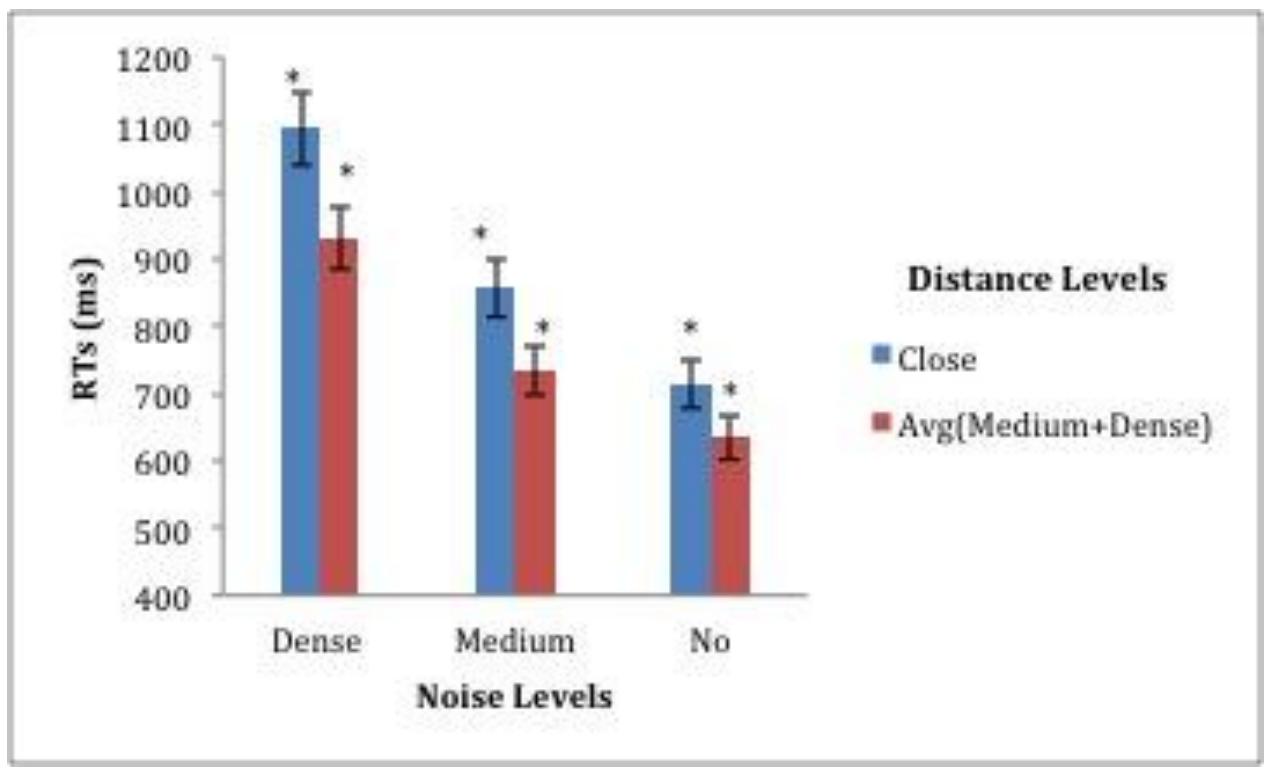

Figure 5: The averaged RT patterns of six subjects. The 72 trials for each condition were repeated over six blocks.

There is a rational relationship between noise manipulation and RT that was predicted from the task design. The greater the degree of noise manipulation, the longer the RTs recorded. Although, in most cases there is a relationship between RT and distance from the reference number of 65 , some participants responded faster to the medium distance numbers than to the far distance numbers, which might answer the question of whether the brain compares the numbers digit by digit or mixes them in a special analog order.

Our block-wise analysis results suggest that the negative peaks represent the end of the learning process. Due to the visual variety of the stimuli, noise manipulations, distance, conditions, and the fact that there were 24 different numbers, the participants had to refresh their memory (re-learning process) three times between measurements. The noise effect was otherwise stable for each block. No-noise and dense noise stimuli produced a negative feedback whereby when the RTs increased for dense noise stimuli, the RTs decreased for nonoise stimuli. The overall conclusion was that visual quality (i.e. noise) was durable during all experiments and increased the RTs.

\section{ERP Results and Discussion}

Brain Vision Analyzer Software Interface (Version 1.05; Brain Products LLC) was used to analyze the EEG data. First, the data was inspected manually to reduce the occurrence of any artifacts. After manual inspection, low $(0.2 \mathrm{~Hz}, 48$ $\mathrm{dB} /$ oct $)$ and high $(30 \mathrm{~Hz}, 48 \mathrm{~dB} / \mathrm{oct})$ pass filters were used to eliminate all noise and artifacts.

After filtering, the data were segmented globally followed by ocular correction of eye blinks. The artifact rejection algorithm was then run for the second noise elimination step, followed by a stimulus classification algorithm. The baseline correction algorithm adjusted the DC offset values. Finally, the segments were averaged to reduce the lack of information and/or any overlapping information.

Figure 6 and 7 illustrates the noise modulation conducted over the two-digit Arabic numbers. As suggested in the literature (Dehaene, 1996; Pinel, et al., 2001; Turconi, et al., 2004), the number comparison effect was reported over the occipital and parietal electrodes along with their frontal counter-part electrodes. 


\section{Close}

Medium

Far
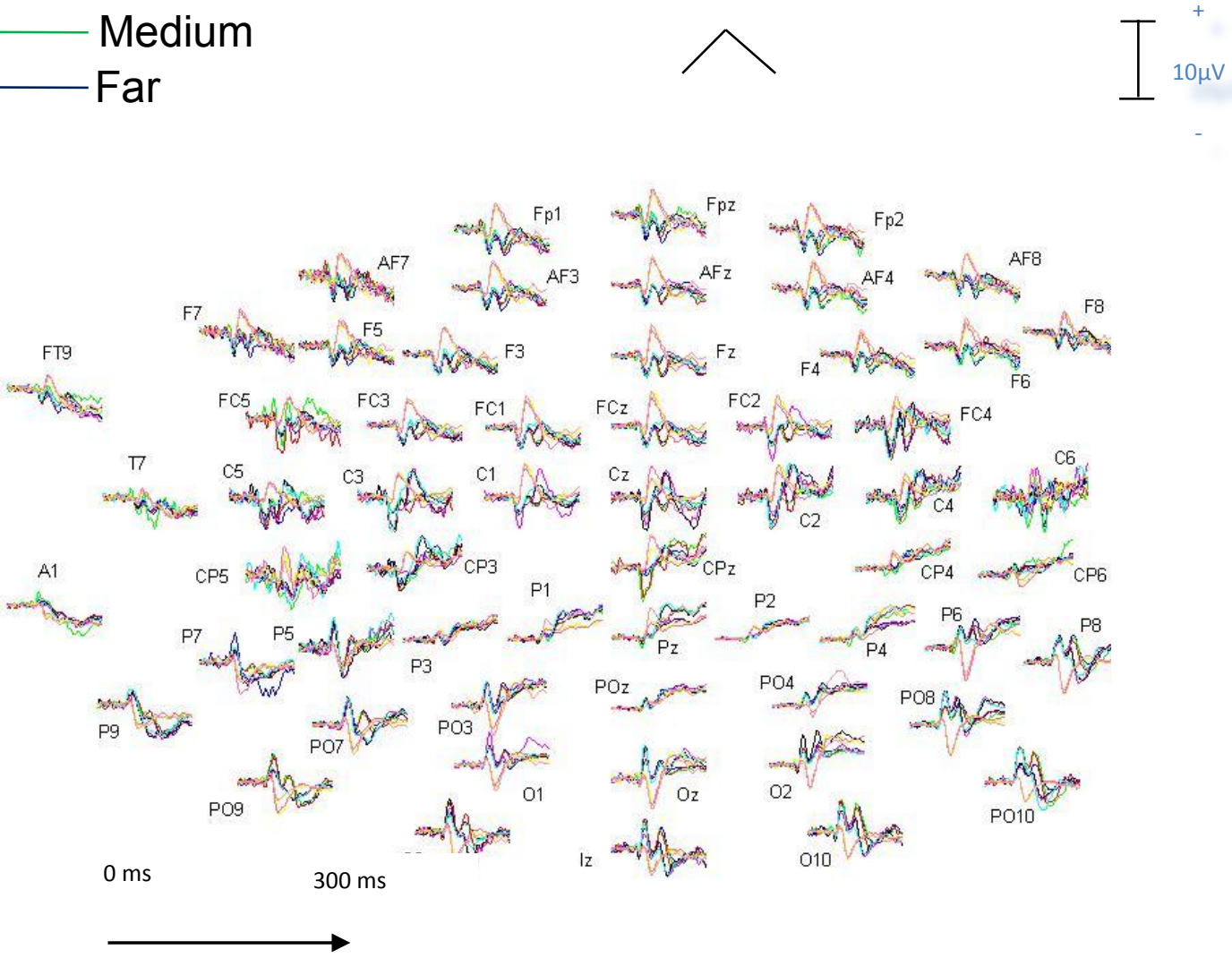

Figure 6: The topographical representations of temporal ERPs of the pilot study participants. The vertical axis is the amplitude value in microvolt and horizontal axis is time in $\mathrm{ms}$ ( $50 \mathrm{~ms}$ before and $600 \mathrm{~ms}$ after the stimulus onset).

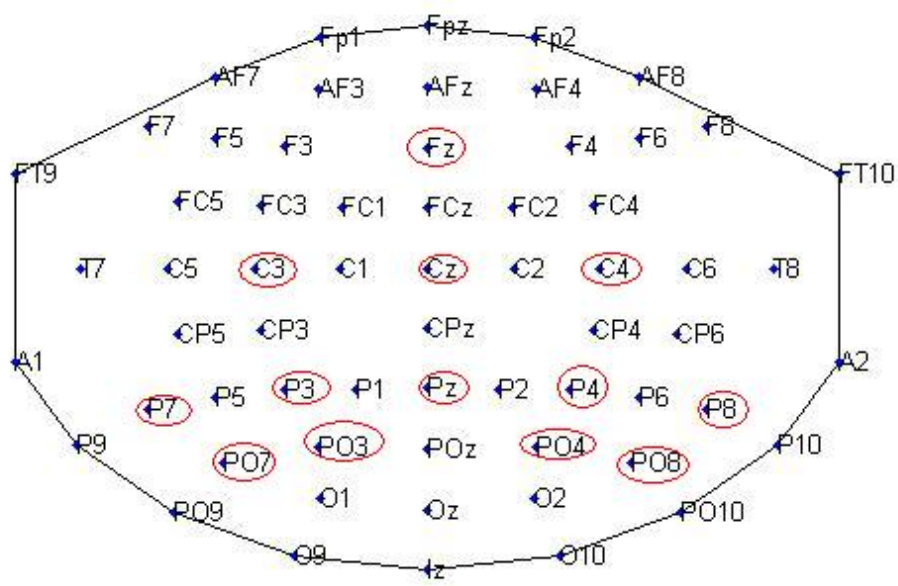

Figure 7: Electrode locations. The electrodes included in the statistical analysis are circled. 
The preliminary results of the project validated the model and design parameters. The visual noise (numerosity), distance modulation and their interaction were consistent for four pilot data. The ERP components will be discussed for the final project to understand the temporal dynamics and underlying mechanisms of particular number comparison tasks. P1 is the earliest positive peak, suggesting an attentional brain activity and luminance discrimination. The main effect of noise was significant in the window of 110 and $130 \mathrm{~ms}$ after the stimulus onset-time $(\mathrm{F}(2,20)=5.5, \mathrm{p}<0.05)$. Post hoc analysis reported a significant difference between no noise stimuli and dense noise stimuli $(\mathrm{F}(1,10)=4, \mathrm{p}<0.05)$ whereas there was no significant difference between dense and medium noise stimuli ( $\mathrm{F}<1, \mathrm{p}>0.05) . \mathrm{P} 2 \mathrm{p}$ occurred only for the noise added stimuli. The second statistical significant for noise occurred in the window of 170-210 ms $(F(2,20)=20$, $\mathrm{p}<0.05)$. A deep negative peak was reported as N1 for the nonoise stimuli. The N1 window was not large for the noise added stimuli. N2 was negativity recorded after P2p for the noise added stimuli. The motor preparation components of ERP followed $\mathrm{N} 1$ for the no noise stimuli, whereas it followed N2 for the noise added-stimuli. The main distance effect was reported in the windows of $330-350 \mathrm{~ms}$ as well as the window of $490-510 \mathrm{~ms}(\mathrm{~F}(2,20)=3.65, \mathrm{p}<0.05, \mathrm{~F}(2,20)=4$, $\mathrm{p}<0.05$, respectively). There was no significant difference between medium and far distance stimuli $(\mathrm{F}<1, \mathrm{p}>0.05)$, however, there was a significant difference between close and medium number stimuli $(\mathrm{F}(1,10)=6.3, \quad \mathrm{p}<0.05)$. The interaction between noise and distance was reported in the windows between 350 and $510 \mathrm{~ms}(\mathrm{~F}(4,40=7.5, \mathrm{p}<0.05)$.

\section{Conclusion}

The present manuscript included the task development phase and testing, as well as the recording system. It was determined that the fixation asterisk provided a more accurate fixation for the participants on the stimulus screen. Similar to the studies in the literature a fixation cross recalled the addition and affected participants' performance (Butterworth, Zorzi, Girelli, \& Jonckheere, 2001; Campbell, 1994; Charras, Brod, \& Lupianez, 2012; Restle, 1970). The visual quality level and the noise type were defined in this manuscript. A very common visual noise type known as "salt and pepper noise" was used to manipulate the number stimulus. The three levels of visual quality were chosen according to the participants' reports and the RTs as being $0 \%$ for no-noise, $60 \%$ for medium and $75 \%$ for dense noise stimuli. It was reported that different spatio-temporal patterns over the parietal lobes represented the visual quality, quantity and their influential relationship. Therefore, we decided to discuss this in terms of the parietal, occipital along with posterior central electrodes.

\section{Future Works}

We finalized our recordings with 15 subjects. We have been currently working on different analysis techniques such as principal component analysis (PCA) and independent component analysis (ICA) to obtain the ERP components from the correlational matrix of the ERP dataset (Handy, 2004; Szucs \& Csepe, 2005). PCA decomposes the multichannel ERP data into a set of orthogonal basis functions, whereas ICA separates the data probabilistically, evaluating the independence of the decomposed components (Handy, 2004). These two approaches, PCA and ICA, will be used to cluster the electrode activities recorded during a specific event. This is suggested as an alternative to the more common clustering and topographic ERP analyzing methods(Murray, Brunet, \& Michel, 2008).

\section{References}

Ballan, M. (2011). Brain dynamics and behavioral basis of a higher level task: VDM Verlag Dr. Müller.

Butterworth, B., Zorzi, M., Girelli, L., \& Jonckheere, A. R. (2001). Storage and retrieval of addition facts: the role of number comparison. $Q J$ Exp Psychol A, 54(4), 10051029.

Campbell, J. I. (1994). Architectures for numerical cognition. Cognition, 53(1), 1-44.

Charras, P., Brod, G., \& Lupianez, J. (2012). Is $26+26$ smaller than $24+28$ ? Estimating the approximate magnitude of repeated versus different numbers. Atten Percept Psychophys, 74(1), 163-173.

Cipolotti, L., Warrington, E. K., \& Butterworth, B. (1995). Selective impairment in manipulating Arabic numerals. Cortex, 31(1), 73-86.

Cohen Kadosh, R., Cohen Kadosh, K., Kaas, A., Henik, A., \& Goebel, R. (2007). Notation-dependent and -independent representations of numbers in the parietal lobes. Neuron, 53(2), 307-314.

Cohen Kadosh, R., \& Henik, A. (2006). A common representation for semantic and physical properties: a cognitive-anatomical approach. Exp Psychol, 53(2), 8794.

Dehaene, S. (1992). Varieties of numerical abilities. Cognition, 44(1-2), 1-42.

Dehaene, S. (1996). The organization of brain activations in number comparison: Event-related potentials and the additive-factors method. Journal of Cognitive Neuroscience, 8(1), 47-68.

Dehaene, S., \& Akhavein, R. (1995). Attention, automaticity, and levels of representation in number processing. $J$ Exp Psychol Learn Mem Cogn, 21(2), 314-326.

Foltz, G. S., Poltrock, S. E., \& Potts, G. R. (1984). Mental comparison of size and magnitude: size congruity effects. J Exp Psychol Learn Mem Cogn, 10(3), 442-453.

Ganor-Stern, D., Tzelgov, J., \& Ellenbogen, R. (2007). Automaticity of two-digit numbers. $J$ Exp Psychol Hum Percept Perform, 33(2), 483-496.

Handy, T. (Ed.). (2004). Event related potentials: MIT Press.

Henik, A., \& Tzelgov, J. (1982). Is three greater than five: the relation between physical and semantic size in comparison tasks. Mem Cognit, 10(4), 389-395.

Kaufmann, L., Koppelstaetter, F., Delazer, M., Siedentopf, C., Rhomberg, P., Golaszewski, S., et al. (2005). Neural correlates of distance and congruity effects in a numerical Stroop task: an event-related fMRI study. Neuroimage, 25(3), 888-898.

McCloskey, M., Caramazza, A., \& Basili, A. (1985). Cognitive mechanisms in number processing and calculation: evidence from dyscalculia. Brain Cogn, 4(2), 171-196.

McCloskey, M., Macaruso, P., \& Whetstone, T. (1992). The functional architecture of numerical processing 
mechanisms:Defending the moduler model. Amsterdam: Elsevier.

Moyer, R. S., \& Landauer, T. K. (1967). Time required for judgements of numerical inequality. Nature, 215(5109), 1519-1520.

Murray, M. M., Brunet, D., \& Michel, C. M. (2008). Topographic ERP analyses: a step-by-step tutorial review. Brain Topogr, 20(4), 249-264.

Noel, M. P., \& Seron, X. (1995). Lexicalization errors in writing arabic numerals: a single-case study. Brain Cogn, 29(2), 151-179.

Nuerk, H. C., Weger, U., \& Willmes, K. (2001). Decade breaks in the mental number line? Putting the tens and units back in different bins. Cognition, 82(1), B25-33.

Pinel, P., Dehaene, S., Riviere, D., \& LeBihan, D. (2001). Modulation of parietal activation by semantic distance in a number comparison task. Neuroimage, 14(5), 10131026.

Restle, F. (1970). Speed of Adding and Comparing Numbers. Journal of Experimental Psychology, 83(2), 274-\&.
Szucs, D., \& Csepe, V. (2005). The effect of numerical distance and stimulus probability on ERP components elicited by numerical incongruencies in mental addition. Brain Res Cogn Brain Res, 22(2), 289-300.

Turconi, E., Jemel, B., Rossion, B., \& Seron, X. (2004). Electrophysiological evidence for differential processing of numerical quantity and order in humans. Brain Res Cogn Brain Res, 21(1), 22-38.

Tzelgov, J. (1997). Specifying the relations between automaticity and consciousness: a theoretical note. Conscious Cogn, 6(2-3), 441-451.

Tzelgov, J., Henik, A., \& Berger, J. (1992). Controlling Stroop effects by manipulating expectations for color words. Mem Cognit, 20(6), 727-735.

Tzelgov, J., Yehene, V., Kotler, L., \& Alon, A. (2000). Automatic comparisons of artificial digits never compared: learning linear ordering relations. $J$ Exp Psychol Learn Mem Cogn, 26(1), 103-120. 